\title{
CLOUD POINTS: CAN WE MEASURE OR MODEL THEM?
}

\author{
Ahmed Hammami and John Ratulowski \\ Oilphase-DBR.,A Schlumberger Company \\ 9419-20 Avenue, Edmonton, Canada T6N 1E5 \\ ahammami@dbrgroup.com
}

João A. P. Coutinho

Departamento de Química da Universidade de Aveiro, 3810 Aveiro, Portugal.

jcoutinho@dq.ua.pt

\begin{abstract}
The terminology "cloud point" has been traditionaly adopted by the American Society for Testing and Materials (ASTM) D2500 to refer to the temperature at which "cloudiness" of a relatively transparent petroleum fluid is observed upon cooling. The cloudiness forms as a "finite and detectable" amount of wax and/or paraffins precipitates from the test fluid contained in a 11/2-in $(\sim 30$ $\mathrm{mm}$ ) diameter glass tube. Nowadays, more sophisticated techniques of differing measurement principles and varying degrees of sensitivity have been developed to measure cloud points of petroleum fluids including opaque hydrocarbon systems (dark oils). Alternative terminologies like wax appearance temperature (WAT) and wax precipitation temperature (WPT), amongst a few others, have also emerged and are used interchangeably. Meanwhile, numerous characterization and modeling efforts have been made to simulate "measured" cloud points of petroleum fluids. The goals of this paper are to provide an objective review of the concept of "cloud point" and to illucidate the adequacy and limitations of the measurement techniques and modeling approaches.
\end{abstract}




\section{INTRODUCTION}

Most crude oils consist of various fractions of heavy hydrocarbons, which are known to precipitate as paraffin deposits mainly due to either evaporation of volatile light ends which otherwise act as naturally occurring solvents or drop in system temperature (Svetgoff, 1984). Accumulation of these solids in transport pipes and process equipment is an old and expensive problem in the petroleum industry. A vital step towards treating this problem is to be able to measure and possibly predict the equilibrium solid-liquid phase boundary (also known as the cloud point locus) as well as the amount of wax deposited under typical process conditions (Hammami and Mehrotra, 1992).

\section{GENERAL CONSIDERATIONS}

The measurement of cloud point depends on a number of factors including oil composition, thermal history, pressure, shear environment, measurement technique, and cooling rate (Hammami and Raines, 1999). An understanding of the key parameters affecting wax precipitation is critical to the interpretation of both laboratory results and model predictions.

\section{Deposition vs. Precipitation:}

The term deposition has often been used to describe the precipitation process. It is important to clarify the difference between the two. While the precipitation may be defined as the formation of a solid phase out of a liquid 
phase, deposition can be described as the formation and growth of a layer of the precipitated solid on a surface. Further, a necessary but not a sufficient condition for deposition is the precipitation of a solid phase from liquid solution. That is, precipitation although a precursor to deposition, does not necessarily ensure deposition. Moreover, whereas the precipitation is mainly a function of thermodynamic variables such as composition, pressure and temperature, the deposition is also dependent on the flow hydrodynamics, heat and mass transfer, and solid-solid and surface-solid interactions. The issue of deposition is beyond the scope of this manuscript; precipitation is our main focus unless otherwise specified.

\section{Thermodynamic vs. Experimental Cloud Point:}

The thermodynamic cloud point is defined as the highest temperature at which a solid phase (i.e., wax) will exist at a given pressure. It is a point on the solid-liquid phase envelope. Measured cloud point, on the other hand, is the temperature at which a detectable amount of a solid phase forms upon cooling in the time frame of the measurement at a given pressure (Karan et al., 2000; Ratulowski et al., 2000). This temperature is a function of the oil composition, the measurement technique, thermal history, the time of measurement, and the fluid properties relating to crystal nucleation and growth. 


\section{Supercooling vs. Superheating:}

Supercooling arises from the fact that the undercooled wax molecules require nucleation sites to start formation. Nucleation sites may be gas bubbles, inorganic impurities or rough foreign surfaces. If such nucleating agents are not present in the liquid, then supercooling will occur until the temperature drops so low that crystals form spontaneously. Once they start to form in a subcooled liquid they will grow rapidly forming a rather unstable/imperfect solid. Superheating, on the other hand, occurs when heat is supplied to a crystal faster that it can melt/dissolve so that the inner portions of the crystals get heated above the equilibrium solid-liquid temperature before actually melting.

Kruka et al. (1995) have argued that, in crude oils, wax molecules are normally surrounded by infinite number of solvent molecules; thereby, the superheating should be less extensive than the supercooling. Gimzewski and Audley (Gimzewski and Audley, 1993) have reported that at the same rate of temperature change, the amount of superheating during melting/dissolution is considerably less than the amount of supercooling during crystallization (Gimzewski and Audley, 1993). Consequently, melting or dissolution temperatures are usually accepted as being much better measure of the equilibrium solid-liquid coexistence temperatures. Ideally, the onset of crystallization will occur at the same temperature as the termination of the melting/dissolution process if the cooling and heating rates are sufficiently low. 
However, even at the scan rate of $0.01^{\circ} \mathrm{C} / \mathrm{min}$, the disparity is reported to be quite large $\left(\sim 3-4^{\circ} \mathrm{C}\right)$ (Gimzewski and Audley, 1993).

\section{Compositions of Petroleum Fluids and Waxes:}

Recognizing that the cloud point is sensitive primarily to the waxy crude composition, it is useful to briefly review the compositions of petroleum fluids and waxes. In general, petroleum fluids can be classified into four major fractions denoted as saturates, aromatics, resins, and asphaltenes. Saturates are non-polar and consist of normal alkanes ( $n$-paraffins), branched alkanes (iso-paraffins) and cyclo-alkanes (naphthenes). Examples of each of these classes of chemicals along with the aromatics, resins and asphaltenes have been reported elsewhere (McCain., 1990; Cruse et al., 1960; Lira-Galeana et al., 2000). Saturates are the largest single source of hydrocarbon or petroleum waxes, which are generally classified as paraffin wax, microcrystalline wax and/or petrolatum (Warth, 1956). Of these, the paraffin wax is the major constituent of most solid deposits from crude oils.

Paraffin waxes are essentially mixtures of long-chain $n$-paraffins, $\mathrm{C}_{\mathrm{n}} \mathrm{H}_{2 \mathrm{n}+2}$ or just $C_{n}$ herein. with carbon chain lengths ranging from $C_{15}$ to $C_{75+}$ (Allen et al., 1989, Srivastava et al., 1993; Hunt, 1996). Asphaltenes and residual oil components often co-precipitate with the paraffin waxes and result in varying appearance (color) and texture to the precipitated solids. As would be expected, waxes from condensates and wet gases tend to be cleaner and lighter in color than those from heavier crudes. The predominately waxy character of the precipitated 
solids can only be defined based on analysis of the deposit and the remedial techniques that can be used to re-dissolve such a solid. In general, only small amounts of aromatic components co-precipitate with waxes and the resulting solid material usually melts by applying heat. It is interesting to note that petroleum wax crystal size decreases as its composition becomes more complex. Typical wax crystals precipitated from real crude oils are indeed much smaller than those crystallized from “model” solvents or simple mixtures (Anderson et al., 2001).

Analytical methods have been available for some time for the separation and characterization of asphaltenes in heavy crudes and of waxes in waxy crudes (Fuhr et al., 1999). A review of these techniques is reported elsewhere (LiraGaleana and Hammami, 2000). Increasingly both asphaltenes and waxes are being found in the same crudes, and the established methods for their separation are often not applicable. Fuhr et al. (1999) developed a method for the analytical separation of asphaltenes and waxes when they co-exist in significant amounts in the same crudes. Typical wax and parent oil analyses usually involve total wax content quantification, n-paraffin carbon distribution to the highest attainable carbon number, wax appearance temperature and/or solid-liquid equilibria wax cut curve (Lira-Galeana and Hammami, 2000). Examples high-temperature gas chromatographs (HTGC) of a wax deposit and its parent oil are shown in Figure 1.

As expected, the heavier n-paraffin molecules are significantly more concentrated in the wax deposit than in the parent oil. The observed humps 
beneath the patterns of $n$-paraffins peaks are presumably ascribed to the presence of branched and cyclic paraffins as well as aromatics (Fuhr et al., 1999). The nparaffin peaks at retention times below 20 min are clear indication of the presence of trapped/occluded oil in the wax deposit.

It is conceivable that the heaviest n-paraffin present in the oil has a dramatic effect on the cloud point and the higher the paraffin decay (defined as the ratio $\left.C_{n} / C_{n+1}\right)$ is, the more difficult the detection of the true cloud point that lies at the end of a very long and thin tail will be.

\section{Mechanism of Paraffin Wax Crystallization:}

Paraffin waxes are semi-crystalline in nature and tend to crystallize/precipitate from crude oils at and below the equilibrium solid-liquid temperature (thermodynamic cloud point). Crystallization is the process whereby an ordered solid structure is produced from a disordered phase, such as a melt or dilute solution (e.g. crude oil). It usually involves two distinct stages, namely nucleation and growth which may be considered separately. These are described in earlier publication (Hammami and Raines, 1999).

Figure 2 depicts three idealized models of paraffin wax crystallization, namely (a) $n$-paraffins only, (b) $n$-paraffins + iso-paraffins and (c) $n$-paraffins + iso-paraffins + cyclo-paraffins, to illustrate the effect of molecular structure and geometry on the nucleation process and the resulting crystal stability. As can be seen in Figure 2 (a), the $n$-paraffins are flexible hydrocarbon molecules and, hence, 
tend to align/cluster together upon cooling and precipitate from crude oil as "stable" wax solids. The iso-paraffins are also flexible hydrocarbon molecules; however, the corresponding side chains tend to delay the formation of wax nuclei (i.e., depress the cloud point) and usually form unstable wax solids (crystals with defects) as shown in Figure 2 (b). Cyclo-paraffins, also known as naphthenes, are stiff (least flexible) and bulky in nature; they tend to disturb and/or disrupt the wax nucleation and growth processes. The corresponding wax crystals are the least stable saturates (of the microcrystalline wax type) as depicted in Figure 2 (c).

It is conceivable that most commercial wax inhibitors, which are polymeric in nature, are specifically synthesized to be branched and/or contain some steric functional groups to help interfere with the paraffin nucleation process and promote crystal imperfections; hence, less stable and easy to dissolve (low heat of fusion) wax crystals are formed. The nucleation temperature and, in turn, cloud point would be expected to shift further to the lower temperature scale in the presence of low molecular weight paraffins (i.e., light ends) and aromatics which are known to be adequate solvents for wax. Whereas, resins do not usually play a significant role on wax precipitation, the presence of impurities and/or amorphous organic solids (such as asphaltenes) in the oil usually induce wax nucleation process as they tend to lower the energy barrier for forming the critical wax nucleus (Hammami and Raines, 1999). 


\section{CLOUD POINT MEASUREMENT TECHNIQUES}

In the open literature, most of the laboratory measurements of the cloud points are made on stock tank oil (STO) samples. These measurements are sensitive to the thermal history as well as the experimental technique used in the laboratory such as, the standard ASTM D2500-88 or IP 219/82 methods, differential scanning calorimetry (DSC), cross polar microscopy (CPM), light transmittance (LT), viscometry, cold finger (CF), filter plugging (FP), and Fourier Transform (FT-IR) spectroscopy, to name a few. As such, the true definitions depend on the measurement principles of the equipment and techniques/procedures being employed (Lira-Galeana et al., 2000; MongerMcClure et al., 1999).

For instance, the ASTM methods rely on visual observation of the wax crystals; they require the liquids to be transparent in layers about $30 \mathrm{~mm}$ in thickness and, hence, often cannot be used to test dark crude oils (e.g. black oils). For black oils, WAT are usually inferred from viscometry, DSC, LT or CPM. While the first three techniques require that sufficient paraffin crystallize before the properties of the crude oil change enough for the liquid/solid (L/S) phase transition to be detected, CPM allows for direct visual observation at the microscopic level (crystal size of the order of $1 \mu \mathrm{m}$ ) of the WAT. In general, CPM has been found to be more sensitive than other techniques for detecting crystalline wax deposits. That is, the limit of detection of the DSC and LT techniques is larger and cloud point values from these techniques will lie further 
inside the solid-liquid phase envelope. It is also possible that for low wax content crude oils, WAT and/or the presence of paraffin crystals may go completely undetected by viscometry, DSC and/or LT, leaving the investigator unaware of potential waxing problems. The CPM is generally deemed to be the most sensitive and the only direct visual technique applicable for all non-biodegraded oils including opaque fluids (Hammami et al., 1999; Erickson et al., 1993).

The problem with cloud point measurement techniques is that no technique available seems to be able to measure the very first crystal (solid nucleus) to appear in a representative fluid sample under true thermal equilibrium. All techniques generally require that some amount of solid wax come out of solution to produce a detectable signal. Several techniques use temperaturescanning rates that prevent the sample from being in equilibrium thus producing a further error. Finally some techniques are indirect and often produce signals which interpretation can be highly subjective.

The wax crystallization rate (i.e., kinetics of precipitation) is perhaps the most critical parameter that greatly contributes to the uncertainties of cloud point measurements. Kruka et al (1995) have reported that the majority of the available measurement techniques yield cloud points comparable to within $\pm 3^{\circ} \mathrm{C}$ for oil samples that precipitate large quantities of wax rapidly (i.e., precipitation rate on the order of $\sim 0.4$ mass $\% /{ }^{\circ} \mathrm{C}$ ). In most cases of Gulf of Mexico oils, however, the wax precipitation rate is reported to be an order of magnitude smaller than 
indicated above. For such oils, the cloud point determination is rather tricky and strongly dependent on the measurement technique employed (Kruka et al., 1995).

Ratulowski et al (2000) have used a cold finger technique to measure clould points of various crude oils including biodegraded oils. Recognizing the aforementioned kinetic limitations of wax precipitation, they have adopted two "deposition" test procedures, namely a standard and an extended tests. The standard test lasts for 2 hours; the corresponding cloud point correlates to the temperature at which a fixed mass $\%$ solid is precipitated out of solution at equilibrium. The extended test runs for 24 hours, and it correlates to the temperature at which a fixed mass $\%$ solid (lower than the standard test) is precipitated out of solution at equilibrium. Based on an extensive testing program, Ratulowski et al (2000) have concluded that the kinetics associated with different fluid properties play less of a role in the longer tests. They argued that the correlated mass $\%$ out of solution should approach the limit of detection of the technique as the test time becomes large.

In short, one must recognize that cloud points are often measured during cooling experiments. Not only this property is dependent on the technique (sensitivity) used but also on the wax concentration, paraffin distribution, kinetics of wax precipitation, cooling rate (the faster the cooling rate, the more depressed the cloud point would be due to supercooling effects), and the thermal history of the waxy crude. It is therefore critical to fully characterize waxy deposits and/or parent oils to gain insights into the concentration and distribution of the active 
wax components (n-paraffins) as well as the kinetics of wax precipitation. It is also useful to measure the wax dissolution temperature at sufficiently low heating rate $\left(<1^{\circ} \mathrm{C} / \mathrm{min}\right)$ at least in addition to the wax appearance temperature. It has been established that when crystalline materials are heated slowly, the temperature at which the last traces of crystallinity disappear is well defined, reproducible and independent of any previous thermal history of the sample (Hammami et al., 1992; Wunderlich, 1976). Such a temperature is deemed the one of thermodynamic significance, for it is the temperature at which the most perfect crystallites are unstable relative to the pure melt/solution; hence, can be identified with the equilibrium "cloud point".

\section{THERMODYNAMIC MODELS FOR PETROLEUM WAX}

The modelling of wax formation in petroleum fluids is based in the thermodynamic description of the equilibrium between the solid wax and the crude liquid phases. The thermodynamic description is done for each compound involved in the equilibrium by the general Solid-Liquid Equilibrium equation that relates the composition in both phases with the non-ideality of the phases and the pure component thermophysical properties (Prausnitz et al., 1999):

$$
\ln \frac{s \gamma^{s}}{x \gamma^{l}}=\frac{\Delta h_{m}}{R T_{m}}\left(\frac{T_{m}}{T}-1\right)+\frac{\Delta h_{t}}{R T_{t}}\left(\frac{T_{t}}{T}-1\right)-\frac{\Delta C p_{m}}{R}\left(\frac{T_{m}}{T}-\ln \frac{T_{m}}{T}-1\right)
$$

The parameters of Equation (1) are defined elsewhere (Prausnitz et al., 1999). 
This equation is the base for all the models reported in the literature. Their differences arise in the approach to the description of the non-ideality of the solid and liquid phases. For low pressure systems (dead oils), the liquid phase can be considered ideal (Erickson et al., 1993; Coutinho et al., 2001) or may be described using a free energy model (Won, 1986; Hansen et al., 1988; Coutinho et al., 1996; Lira-Galena et al., 1996). For high pressure systems (live oils), on the other hand, the liquid phase is generally described using an Equation of State (Lira-Galena et al., 1996; Pan et al., 1997; Pauly et al., 2000).

Meanwhile, the solid phase is commonly described using one of two opposite approaches. Either the authors assume that the solid phase is a sum of independent pure components or pseudo-components (Ratulowski et al., 2000; Lira-Galena et al., 1996; Pan et al., 1997) or that it is made up of a solid solution, stable or not, of all the materials that crystallize. Some authors that consider the formation of a solid solution assume it being ideal (Erickson et al., 1993; Coutinho et al., 2001; Won, 1986; Hansen et al., 1988; Coutinho et al., 1996). Most of them adopt some form of free energy model to describe the non-ideality of the solid phase.

The misty region in most models is in the definition of what is the nature of the solid formed. Pioneer authors generally assume that all compounds present in the mixture eventually become part of the wax phase (Won, 1986; Hansen et al., 1988) This assumption is also adopted in recent publications (Lira-Galena et al., 1996; Pan et al., 1997) in spite of the growing evidence that the wax is made 
up primarily of compounds with long n-alkyl chains. The majority of the nalkanes with carbon number between 18 and 60 are polymorphic and hence assume more than one crystalline form below their melting points. The thermophysical properties associated with polymorphic wax crystals depend on which solid phase is adopted. Most authors do not clearly state which solid phase they are assuming and, hence, do not account for the solid-solid transitions. Strong evidence today shows that the alkyl chains in the wax lead to an orthorhombic structure (Chevallier et al., 2001) even if some isoalkanes or alkyl aromatic compounds are present (Dorset, 2000). The thermophysical properties, when the solid phase is poorly defined, are usually fitted to a set of experimental data and the values adopted, usually to a large extent far from the pure compounds data, actually compensate for weaknesses in the non-ideality description; thereby, are of limited applicability for systems other than those used in the development of the correlation. Only few authors clearly state the solid phase adopted, the compounds present in the solid phase and use thermophysical properties derived from the pure compounds thermophysical data (Ratulowski et al., 2000; Coutinho et al., 2001; Coutinho et al., 1996).

Although many of the above models claim some predictive capability, most are fitted to experimental data and all are validated with experimental data. The data sets that are typically available for crude oil systems are either cloud points or wax cut curves. Unfortunately, some researchers have assumed that measured cloud points are the thermodynamic phase boundary and that literature 
repoted wax cuts contain no occluded oil. These assumptions invalidate the tuning and validation procedures and lead to models with little real predictive capability. Thermodynamic models should be validated or tuned to equilibrium data. While the thermodynamic phase boundary in crude oil systems is not measurable, good equilibrium data can be readily obtained. Phase amount and compositions from carefully performed solid-liquid-vapor equilibrium flashes are the most representative data to tune or validate thermodynamic models. A match to these data is illustrated in Figure 2. Once the predictive capability of the model is established with these data, predictions from the model at the measured cloud point temperature and pressure can give some insight into the detection limit and effect of kinetics for the various measurement techniques. Estimates of detection limit range between 0.01 to 0.1 mass $\%$ of solid out of solution for the various techniques discussed above.

In non-biodegraded oils, heavy molecular weight normal paraffins determine cloud points. Therefore, the most successful thermodynamic models for predicting cloud points require a detailed normal paraffin distribution. The largest paraffin present in the oil at a concentration above its solubility threshold for a given temperature sets the thermodynamic phase boundary. The shape of the distribution will influence both the wax cut curve and the temperature difference between the measured cloud point and the thermodynamic phase boundary. This shape is characterized by the decay of the heavy normal paraffin content with increasing carbon number relative to the solubility isotherms. 
The effect of normal paraffin distribution on cloud point measurements and modeling can be illustrated by another observation. Although cloud points for crudes are quite difficult to measure or predict, cloud points for refined products such as jet fuels or diesels are easily detected and modeling of them is quite reliable (Coutinho, 2000). In these systems, the sharp decay of the normal paraffin distribution results in the amount of precipitated solid quickly increasing to detectable levels once the solubility threshold of heavier paraffins is attained. Therefore, measured cloud point and the thermodynamic phase boundary is essentially the same temperature.

In summary, thermodynamic models for cloud point are all based on a crystalline solid model for solid phase fugacities. The models differ in the way in which the liquid phase fugacities are modeled and in the assumptions of the solid phase structure. Models in which the solid phase structure is well defined and true thermo chemical property data are used can be predictive.

\section{CONCLUSIONS}

The distribution of normal paraffins in non-biodegraded oils has a dramatic effect on the wax formation and in particular on the detection of cloud points. Systems with sharply decaying paraffin distributions will precipitate detectable quantities of solids just below the thermodynamic phase boundary. In these cases, measured cloud point corresponds to the thermodynamic phase boundary and is easily identified by most techniques. However, most crude oils 
have a normal paraffin distribution with a high molecular weight tail. Both precipitation kinetics and solid phase detection limits make reliable measurements of the phase boundary impossible for these systems. A carefully performed cloud point measurement at best corresponds to the wax cut at the detection limit of the measurement technique which can be significantly below the temperature of the thermodynamic phase boundary. A detailed compositional analysis coupled with a properly validated thermodynamic model can provide insight on the interpretation of cloud points measured using different techniques. Future research activities must focus on improving existing and/or developing new techniques to detect and quantify, in addition to the onset of wax precipitation, the associated liquid-solid phase transformation kinetics. These latter effects must be captured and/or incorporated into the mathematical descriptions of the wax crystallization process. 


\section{REFERENCES}

Allen, T. O. and Roberts, A. P., 1989. Production Operations: Well Completions, Workover, and Stimulation, second edition, Oil \& Gas Consultants International. Inc. 2: 11.

Anderson, T, Peters, H. S., Torres, R. A., Nagy, N. A., and Schruben, D. L., 2001. Wax crystal size distribution versus composition, Fuel 80:1635.

Chevallier, V., Briard, A.J., Petitjean, D., Hubert, N., Bouroukba, M., and Dirand, M., 2001. Influence of the distribution general shape of n-alkane molar concentrations on the structural state of multi-alkane mixtures Mol Cryst Liq Cryst. 350: 273.

Coutinho J.A.P., 2000. A Thermodynamic model for predicting wax formation in jet and diesel fuels. Energy and Fuels, 14: 625

Coutinho J.A.P. and Daridon, J.L., 2001. Low-Pressure Modeling of Wax Formation in Crude Oils, Energy and Fuels, 15: 1454.

Coutinho, J.A.P., Stenby, E.H., 1996. Predictive local composition model for solid-liquid and solid-solid in n-alkenes: Wilson equation for multicomponent systems. Ind. Eng. Chem. Res. 35: 918

Cruse, W. A. and Stevens, D. R., 1960 in: Chemical Technology of Petroleum, McGraw-Hill Book Publishers Inc., New York City, Chap. XXII.

Dorset, D.L., 2000. Crystallography of Real Waxes: Branched Chain Packing in Microcrystalline Petroleum Wax Studied by Electron Diffraction, Energy and Fuels, 14 (3): 685

Erickson, D. D., Niesen, V. G. and Brown, T. S., 1993. Thermodynamic Measurement and Prediction of Paraffin Precipitation in Crude Oil, SPE 26604 Presented at the 68th Annual Technical Conference and Exhibition of the Society of Petroleum Engineers in Houston TX, October 3-6. 
Fuhr, B. J., Holloway, L. R., and Hammami, A., 1999. Analytical Considerations Related to Asphaltenes and Waxes in the Same Crudes, Energy \& Fuels 13: 336.

Gimzewski, E., Audley, G., 1993. Monitoring wax crystallisation in diesel using differential scanning calorimetry (DSC) and micro-calorimetry, Thermochim. Acta 214 : 149.

Hammami, A, and Mehrotra, A. K., 1992. Non-isothermal crystallization kinetics of binary mixtures of $n$-alkanes: ideal eutectic and isomorphous systems, Fuel 75(4): 500 .

Hammami, A., Raines, M.A., 1999. Paraffin Deposition from Crude Oils: Comparison of Laboratory Results to Field data. SPE J., 4: 1

Hansen, J.H., Fredenslund, Aa., Pedersen, K.S., Rønningsen, H.P., 1988. A thermodynamic model for predicting wax formation in crude oils, AIChE J. 12: 1937.

Hunt, A.., 1996. Uncertainties Remain in Predicting Paraffin Deposition, Oil \& Gas J. 94 (July 29) No. 31: 96.

Karan, K., Ratulowski, J., and German. P., 2000. Measurement of Waxy Crude Properties Using Novel Laboratory Techniques, SPE 62945 Presented at the 75th Annual Technical Conference and Exhibition of the Society of Petroleum Engineers in Houston TX, October 1-4.

Kruka, V. R., Cadena, E. R., and Long, T. E., 1995. Cloud-Point Determination for Crude Oils, JPT (August) 681.

Lira-Galeana, C., Hammami, A., 2000 in: Asphaltenes and Asphalts 2; Yen, T. F., Chilingarian, G. eds.; Elsevier Science Publishers: Holland, Chapter 21.

Lira-Galena, C., Firoozabadi, A., Prausnitz, J.M., 1996. Thermodynamics of wax precipitation in petroleum mixtures, AIChE J., 42: 239 
McCain, W. D. Jr., 1990 in: The Properties of Petroleum Fluids, PennWell Publishing Co., Oklahoma Chap. 1

Monger-McClure, T. G., Tackett, J. E., Merrill, L. S., 1999. Comparison of Cloud Point Measurement and Paraffin Prediction Methods, SPE Prod. \& Facilities, 14: 4.

Pan, H., Firoozabadi, A., and Fotland, P., 1997. Pressure and composition effect on wax precipitation: Experimental data and model results, SPE Production \& Facilities, November: 250.

Pauly, J,. Daridon, J.L., Coutinho, J.A.P., Lindeloff, N., and Andersen, S.I., 2000. Prediction of solid-liquid phase diagrams of light gases-heavy paraffin systems up to $200 \mathrm{MPa}$ using an equation of state- $\mathrm{G}^{\mathrm{E}}$ model, Fluid Phase Equilibria, 167: 145 .

Prausnitz, J.M., Lichtenthaler, R.N., and Azevedo, E.G., Molecular Thermodynamics of Fluid-Phase Equilibria; 3rd ed., Prentice-Hall, Upper Saddle River, NJ (1999).

Ratulowski, J., Westrich, J., Leitko, A., 2000. Thermodynamic Model For Wax Precipitation in Live Oil Systems, Paper presented at ACS Spring National Meeting, San Francisco, March 26-30.

Srivastava, S. P., Handoo, J., Agrawal, K. M., Joshi, G. C., 1993. PhaseTransition Studies in n-Alkanes and Petroleum Related Waxes - A Review, $J$. Phys. Chem. Solids 54: 639.

Svetgoff, J., 1984. Paraffin Problem Can Be Resolved With Chemicals," Oil \& Gas J. (Feb 27) 79.

Warth, A. H., in: The Chemistry and Technology of Waxes, 2nd Edition, Reinhold, New York (1956) Chapter 5. 
Won, K. W., 1986. Thermodynamics for Solid Solution-liquid-vapor equilibrium, wax phase formation from heavy hydrocarbon mixture. Fluid Phase Equilib 30: 265.

Wunderlich, B., 1976. in: Macromolecular Physics, volume 2, Academic Press, New York. 
Figure 1. HTGC chromatograms of (a) Parent dead oil and (b) wax deposit 


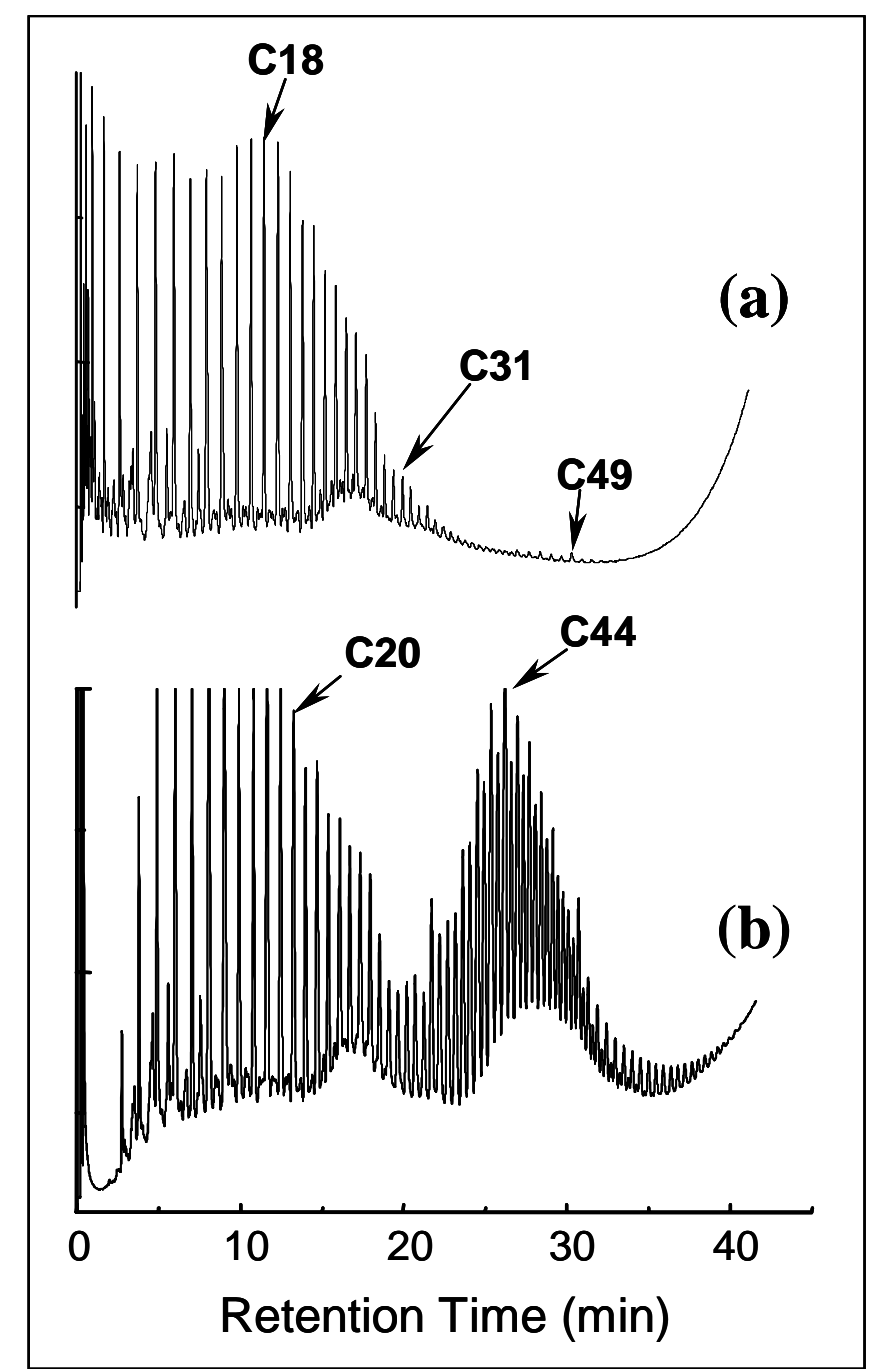


Figure 2. Schematic illustrations of idealized paraffin crystallization models: (a) $n$-paraffins, (b) $n-+i$ so-paraffins and (c) $n-+i$ so- + cyclo-paraffins 

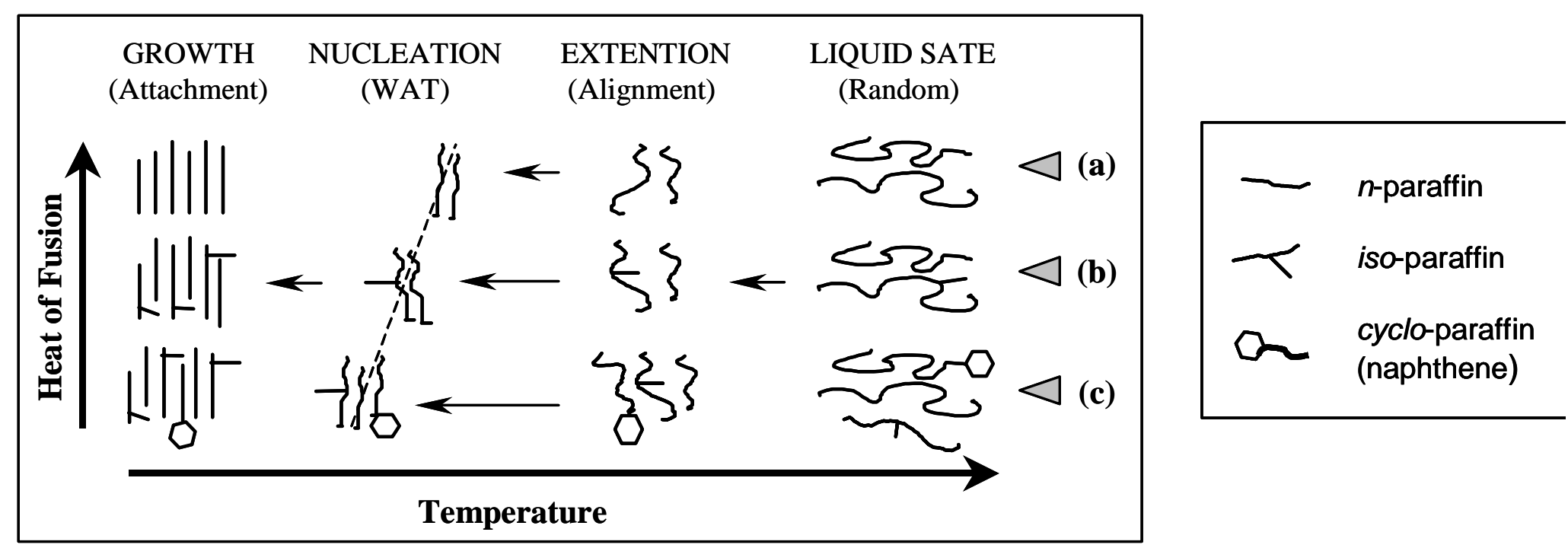
Figure 3: Example of matched experimental data with thermodynamic model results (Ratulowski et al., 2000): (a) Wax cut curve; (b) Carbon number distribution of precipitated wax at $4.5^{\circ} \mathrm{C}$ 


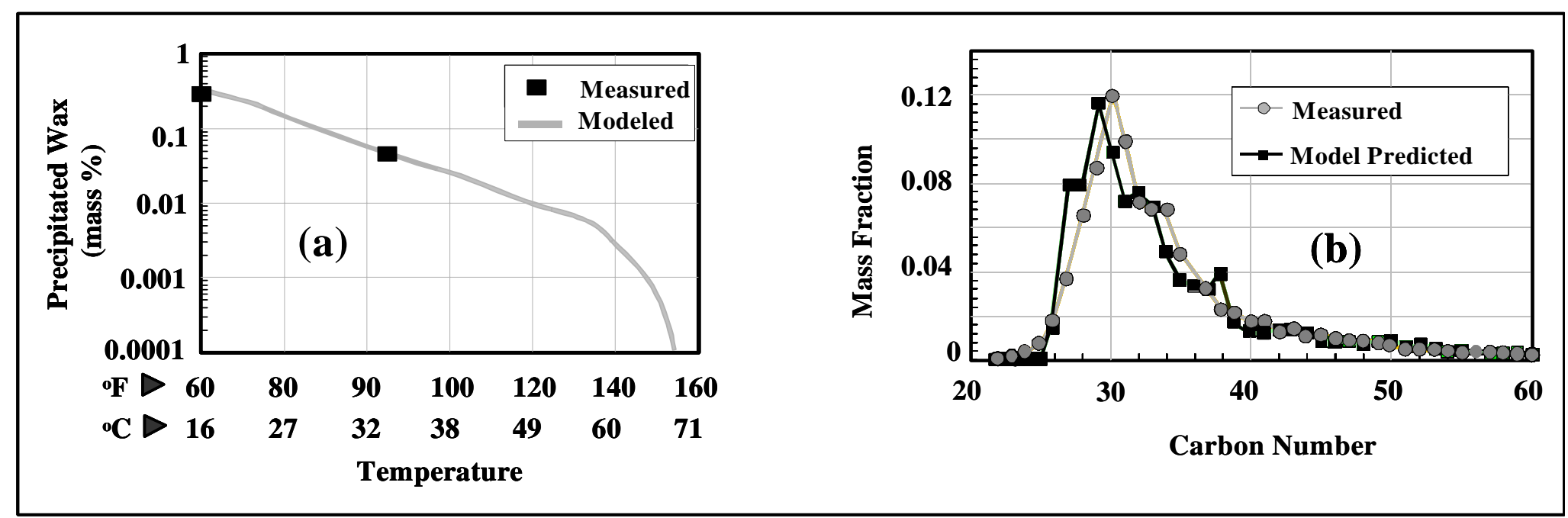

